\title{
BER Performance Analysis of CDMA Reverse Link under AWGN Channel
}

\author{
Sanjeev Kumar \\ AP, Deptt.of ECE, ACET \\ $12 \mathrm{~km}$ stone,Amritsar-Jalandhar, \\ GT road, \\ Amritsar, Punjab, India
}

\author{
Monika Tuteja \\ Lecurer, Deptt. of ECE \\ College of Engineering \& \\ Mangement, \\ Kapurthala, Punjab, India
}

\author{
Ramneek Singh \\ Lecturer, Deptt.of ECE \\ DAV Institute of Engineering \& \\ Technology \\ Jalandhar, Punjab, India
}

\begin{abstract}
The bit error rate (BER) performance of the Code Division Multiple Access (CDMA) cellular system based on IS-95 standard in the presence of an additive white Gaussian noise (AWGN) and interference has been investigated in this paper. The performance is evaluated under two types of decision feedback receivers for the CDMA reverse link. These two feedback receivers are: (a) Hard decision Viterbi decoder in which coded bit is estimated based on Hamming Distance method and (b) Soft decision Viterbi decoder in which Euclidean Distance method is used for coded bit estimation. The comparison of these two techniques of decision feedback receivers of CDMA is done under AWGN channel. The performance of CDMA system is shown in graphs between BER versus Energy per bit to Noise Ratio i.e. Eb/No ratio.
\end{abstract}

\section{Keywords}

CDMA, BER, Chip Rate, Chip period, Eb/No, PN codes.

\section{INTRODUCTION}

Code Division Multiple Access (CDMA) is a digital radio system that transmits streams of bits (PN codes). CDMA permits several radios to share the same frequencies and have a significant economic advantage over TDMA-based standards, or the oldest cellular standards that used frequency-division multiplexing. Its greater bandwidth efficiency and multiple access capabilities make it the leading technology for relieving spectrum congestion caused by the explosion in popularity of cellular mobiles and fixed wireless telephones and wireless data terminals [1].

The IS-95 standard describes a Code Division Multiple Access (CDMA) system in which the data signal is multiplied by a high rate spreading signal. This spreading signal is formed from a pseudo-noise code sequence, which is then multiplied by a Walsh code for maximum orthogonality to the other codes use in that cell. Typically, CDMA pseudo-noise sequences are very long, thereby giving excellent cross correlation characteristics [2].

The IS-95 standards describe an air interface, a set of protocols used between mobile units and the network. It is widely described as a three-layer stack, physical (PHY) layer, Media Access Control (MAC) and Link-Access Control (LAC) sub layers, and call-processing state machine. In IS-95 system forward channel carries information from the base station to the mobile unit whereas the reverse channel carries information from the mobile unit to the base station. The forward channels are between 869 and $894 \mathrm{MHz}$, while the reverse channels are between 824 and $849 \mathrm{MHz}$ [3]. The various Modulation parameters like user data rate, coding rate data repetition rate, PN chip rate etc. for forward \& reverse link channel are given in the table $1 \& 2$ given below:

Table 1. Forward Link Channel Parameters

\begin{tabular}{|l|c|c|c|c|}
\hline \multicolumn{1}{|c|}{ Parameter } & \multicolumn{4}{c|}{ Data Rate(bps) } \\
\hline User data rate & 9600 & 4800 & 2400 & 1200 \\
\hline Coding Rate & $1 / 2$ & $1 / 2$ & $1 / 2$ & $1 / 2$ \\
\hline $\begin{array}{l}\text { User Data } \\
\text { Repetition Period }\end{array}$ & 1 & 2 & 4 & 8 \\
\hline $\begin{array}{l}\text { Baseband coded } \\
\text { Data Rate }\end{array}$ & 19,200 & 19,200 & 19,200 & 19,200 \\
\hline $\begin{array}{l}\text { PN Chips/Coded } \\
\text { Data Bit }\end{array}$ & 64 & 64 & 64 & 64 \\
\hline PN Chip Rate & 1,2288 & 1,2288 & 1,2288 & 1,2288 \\
\hline PN Chips/Bit & 128 & 256 & 512 & 1024 \\
\hline
\end{tabular}

Table 2. Reverse Link Channel Parameters

\begin{tabular}{|c|c|c|c|c|}
\hline Parameter & \multicolumn{4}{|c|}{ Data Rate(bps) } \\
\hline User data rate & 9600 & 4800 & 2400 & 1200 \\
\hline Coding Rate & $1 / 3$ & $1 / 3$ & $1 / 3$ & $1 / 3$ \\
\hline Coded Data Rate & 28,800 & 28,800 & 28,800 & 28,800 \\
\hline $\begin{array}{c}\text { Bits per Walsh } \\
\text { Symbol }\end{array}$ & 6 & 6 & 6 & 6 \\
\hline $\begin{array}{c}\text { Walsh Symbol } \\
\text { Rate }\end{array}$ & 4800 & 4800 & 4800 & 4800 \\
\hline $\begin{array}{c}\text { Walsh Chip Rate } \\
\text { PN Chips/Code } \\
\text { Symbol }\end{array}$ & 307.2 & 307.2 & 307.2 & 307.2 \\
\hline $\begin{array}{c}\text { PN Chips/Walsh } \\
\text { Symbol }\end{array}$ & 256 & 256 & 256 & 256 \\
\hline $\begin{array}{c}\text { PN Chips/Walsh } \\
\text { Chip }\end{array}$ & 4 & 4 & 4 & 4 \\
\hline PN Chip Rate & 1,2288 & 1,2288 & 1,2288 & 1,2288 \\
\hline
\end{tabular}

Most of the related work in CDMA cellular performance has been focused on the reverse channel as in [4] through [6]. While these investigations consider both shadowing and fading effects, they do not incorporate forward error correction in the analysis. The Performance of CDMA using forward error correction with soft decision decoding has been given in [7] through [10]. The 
performance of the forward DS-CDMA channel with power control in a Rayleigh fading channel was investigated in [11]. The effects of lognormal shadowing and Rayleigh fading using forward error correction in the form of Golay codes with hard decision decoding is given in [12] whereas in [13], Nakagami fading channels were considered using a weak Golay Code with hard decision decoding.

\section{CDMA TRANSMITTER \& RECIEVER}

The forward Link transmitter interoperations comprise of convolution encoding and repetition, block interleaving, long PN sequence, data scrambling, Walsh coding and Quadrature modulation. Diagram is shown below in Fig.1.

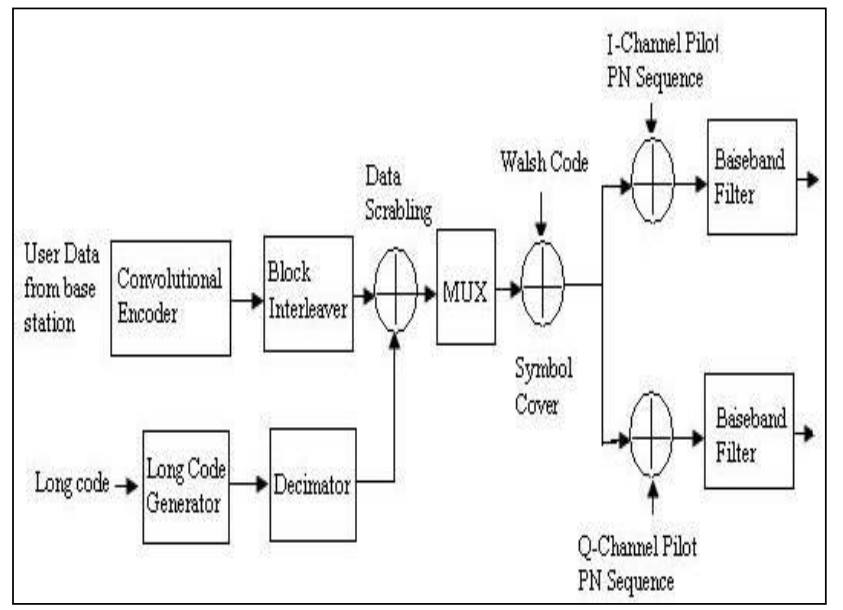

Fig 1: CDMA Forward Link Transmitter Modulation

The processing performed in the terminal receiver of CDMA is being complementary to those of the base station modulation process on the forward Channel and is shown in Fig.2.

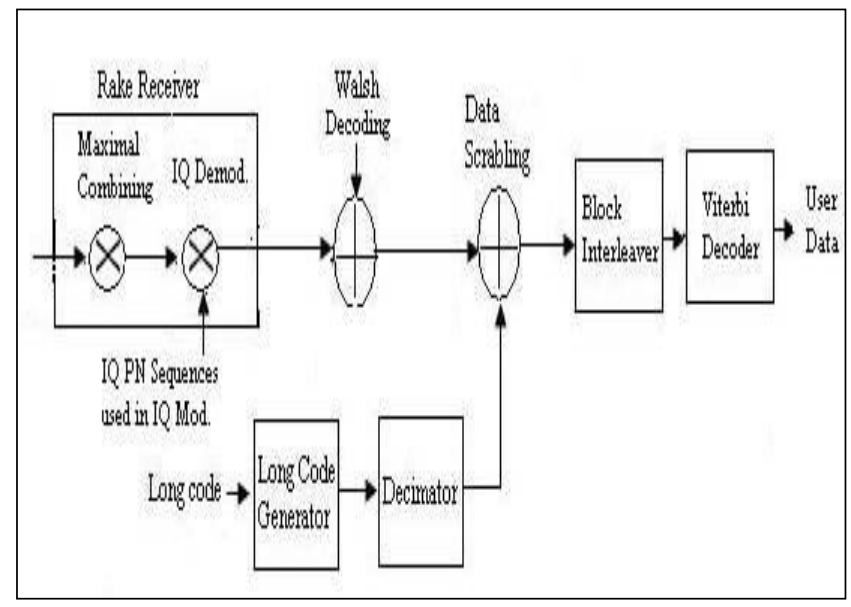

Fig 2: CDMA Reverse Link Receiver Demodulation

The receiver in CDMA used two types of viterbi decoding: Hard decision \& soft decision viterbi decoding.

Soft decision viterbi decoder used euclidean distance method for coding bit estimation. Euclidean distance is found out between the received symbol \& portable transmitted symbol.
The received coded sequence is

$$
\mathrm{y}=\mathrm{c}+\mathrm{n}
$$

Where $\mathrm{c}$ is the modulated coded sequence taking values $+\sqrt{\mathrm{E}}$ if the coded bit is 1 and $-\sqrt{E}$ if the coded bit is $0, n$ is the Additive White Gaussian Noise. The probability distribution function of Soft decision decoding is given as

$$
P(x)=\frac{1}{\sqrt{2 \pi \sigma^{2}}} e^{\frac{-(x-\mu)^{2}}{2 \sigma^{2}}}
$$

With mean $\mu=0$ and variance $\sigma 2=\mathrm{No} / 2$.

The conditional probability distribution function (PDF) of $Y$ if the coded bit is 0 is,

$$
\mathrm{P}(Y / \mathrm{Co})=\frac{1}{\sqrt{\pi \mathrm{No}}} \mathrm{e}^{\frac{-(Y+\sqrt{\mathrm{Ec}})^{2}}{\mathrm{No}}}
$$

Conditional probability distribution function (PDF) of $Y$ if the coded bit is 1 is,

$$
\mathrm{P}\left(Y / \mathrm{C}_{1}\right)=\frac{1}{\sqrt{\pi \mathrm{No}}} \mathrm{e}^{\frac{-(Y-\sqrt{\mathrm{Ec}})^{2}}{\text { No }}}
$$

In soft decision decoding, Euclidean distance is found out between the received symbol \& portable transmitted symbol. Euclidean distance if transmitted coded bit is 0 is,

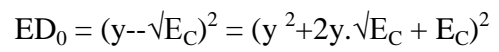

Euclidean distance if transmitted coded bit is 1 is,

$$
E_{1}=\left(y--\sqrt{ } E_{C}\right)^{2}=\left(y^{2}-2 y \cdot \sqrt{E_{C}}+E_{C}\right)^{2}
$$

The terms y2, $\sqrt{E_{C}}$ and $E_{C}$ are common in both the equations they can be ignored. The simplified Euclidean distance is,

$$
\mathrm{ED}_{0}=+\mathrm{y} \text { and } \mathrm{ED}_{1}=-\mathrm{y}
$$

As the Viterbi algorithm takes two received coded bits at a time for processing, we need to find the Euclidean distance from both the bits.

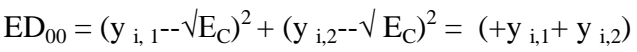

$$
\begin{aligned}
& \mathrm{ED}_{01}=\left(\mathrm{y}_{\mathrm{i}, 1^{--}} \mathrm{V}_{\mathrm{C}}\right)^{2}+\left(\mathrm{y}_{\mathrm{i}, 2^{-}}+\sqrt{ }_{\mathrm{C}} \mathrm{E}^{2}=\left(+\mathrm{y}_{\mathrm{i}, 1}-\mathrm{y}_{\mathrm{i}, 2}\right)\right. \\
& \mathrm{ED}_{10}=\left(\mathrm{y}_{\mathrm{i}, 1}-+\sqrt{ }_{\mathrm{C}_{\mathrm{C}}}\right)^{2}+\left(\mathrm{y}_{\mathrm{i}, \mathrm{2}^{-}}-\sqrt{ }_{\mathrm{C}}\right)^{2}=\left(-\mathrm{y}_{\mathrm{i}, 1}+\mathrm{y}_{\mathrm{i}, 2}\right) \\
& \mathrm{ED}_{11}=\left(\mathrm{y}_{\mathrm{i}, 1^{-}}+\sqrt{ }_{\mathrm{C}}\right)^{2}+\left(\mathrm{y}_{\mathrm{i}, 2^{-}}+\sqrt{ }_{\mathrm{C}}\right)^{2}=\left(-\mathrm{y}_{\mathrm{i}, 1}-\mathrm{y}_{\mathrm{i}, 2}\right)
\end{aligned}
$$


Hard decision viterbi decoding, based on the location of the received coded symbol. Hamming distance is found out between the received symbol \& portable transmitted symbol. Hard decision decoding takes a stream of bits say from the 'threshold detector' stage of a receiver, where each bit is considered definitely one or zero.

\section{SIMULATION RESULTS}

The capacity of CDMA communication system depends heavily on spreading factor and receiver's performance. The receiver performance largely depends on the BER of system. BER is defined as source of performance measurement that specifies the number of bits corrupted or destroyed as they are transmitted from its source to destination. So, in order to improve the performance of system, BER should be minimum. There are several factors that affect BER include bandwidth, SNR, transmission speed and transmission medium. The expression for BER is given as.

$$
\mathrm{BER}=\mathrm{Q}\left[\mathrm{Es} / 2 \sigma \mathrm{n}^{2}\right]^{1 / 2}
$$

Es $=\mathrm{M}^{2}$ of average power of original signals, $\sigma \mathrm{n}^{2}=$ variance of noise in decision variable which may include the AWGN \& processing noise incurred in system.

CDMA IS-95 standard using BPSK modulation in presence of AWGN channel was simulated using MATLAB. The CDMA signal parameters used in simulation are given in table 1 and table 2.The results presented show the BER performance as a function of the channel SNR.

The SNR for each modulation takes into account the number of bits per symbol, and so the signal power corresponds to the energy per bit times the number of bits per symbol. The higher $\mathrm{Eb} / \mathrm{No}$ required for transferring data means that more energy is required for each bit transfer.

As two coded bits are required for transmission each data bit (BPSK), the relation between coded bits to noise ratio Ec/No with bits to noise ratio $\mathrm{Eb} / \mathrm{No}$ is

$$
\mathrm{E}_{\mathrm{c}} / \mathrm{N}_{0}=1 / 2\left(\mathrm{E}_{\mathrm{b}} / \mathrm{No}\right)
$$

The Probability of BER with hard decision and soft decision viterbi decoding are given in figure 3 and figure 4 .

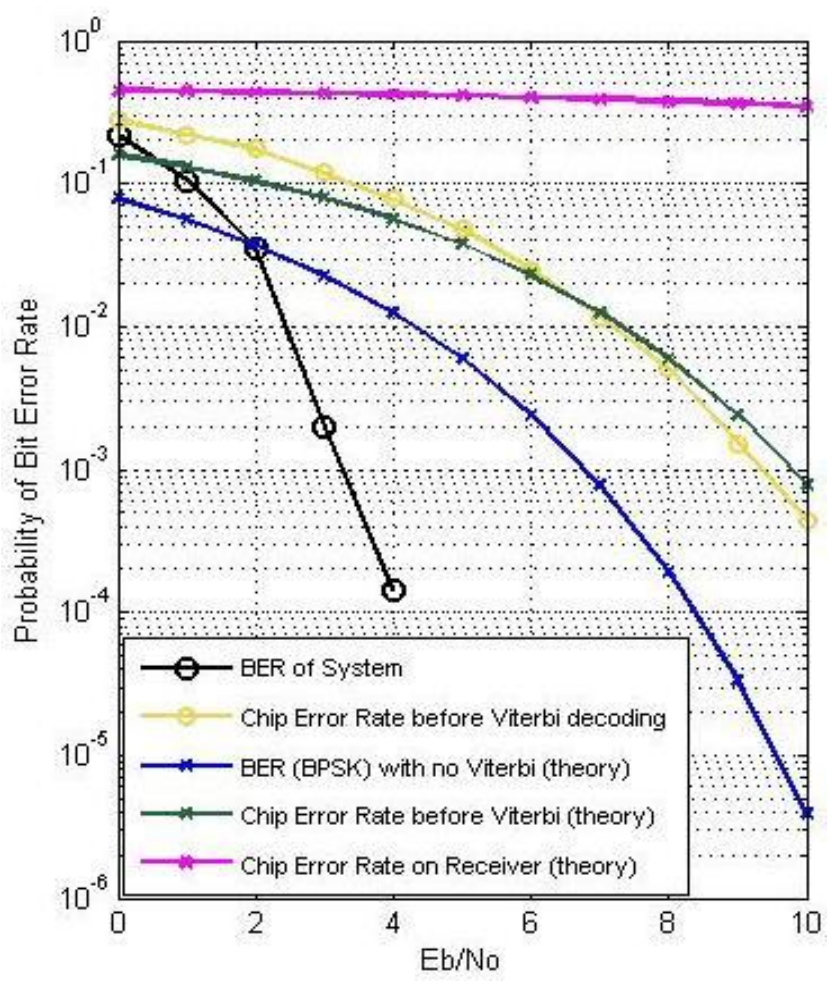

Fig 3: BER Probability with Hard Decision Decoding

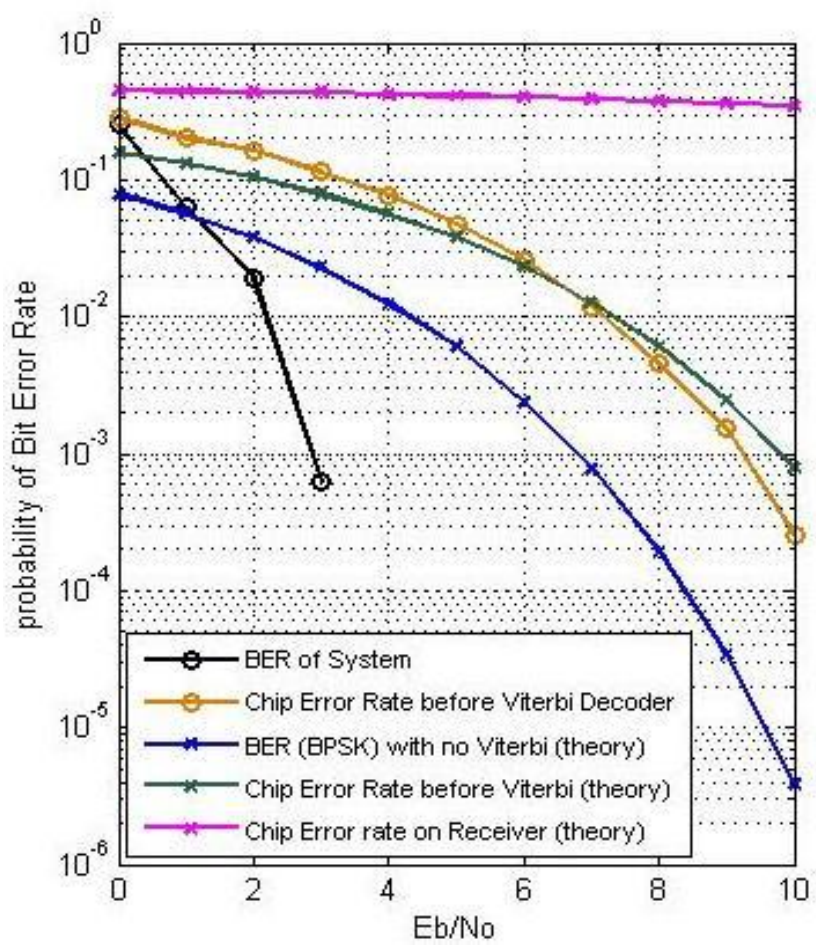

Fig 2: BER Probability with Soft Decision Decoding 


\section{CONCLUSION}

The results shown in figure 3 and figure 4 gives the probability of BER when we use two different viterbi decoding i.e Soft decision and hard decision decoding. It is concluded from results that probability of BER is found to be less with hard decision viterbi decoding as compared with Soft decision viterbi decoding.

\section{REFERENCES}

[1] Andrew J. Viterbi. CDMA: Principles of Spread Spectrum Communication. Addison-Wesley, Reading, MA, 1995.

[2] Qualcomm, "Overview of IS-95 CDMA System" Oct 1996.

[3] Ding, W. and Marchionini, G. 1997 A Study on Video Browsing Strategies. Technical Report. University of Maryland at College Park.

[4] W. Ye and A. M. Haimovich, "Performance of Cellular CDMA with Cell Site Antenna Arrays, Rayleigh Fading, and Power Control Error," IEEE Transactions on Communications, vol. 48, no. 7, pp. 1151-1159, July 2000.

[5] T. T. Tjhung and C. C. Chai, "Distribution of SIR and Performance of DS-CDMA Systems in Lognormally Shadowed Rician Channels," IEEE Transactions on Vehicular Technology, vol. 49, no. 4, pp. 1110-1125, July 2000.

[6] B. Hashem and E. S. Sousa, "On the Capacity of Cellular DS/CDMA Systems under Slow Rician/Rayleigh-Fading Channels," IEEE Transactions on Vehicular Technology, vol. 49 , no. 5 , pp. $1752-1759$, Sep. 2000.

[7] A. J. Viterbi, A. M. Viterbi, E. Zehavi, "Performance of Power-Controlled Wideband Terrestrial Digital Communication," IEEE Transactions on Communications, vol. 41, no. 4, pp. 559-569, Apr. 1993.
[8] R. D. Cideciyan, E. Eleftheriou, M. Rupf, "Concatenated Reed-Solomon/Convolutional Coding for Data Transmission in CDMA-Based Cellular Systems," IEEE Transactions on Communications, vol. 45, no. 10, pp. 1291-1303, Oct. 1997.

[9] A. M. Earnshaw and S. D. Blostein, "A Combined SoftDecision Deinterleaver/Decoder for the IS95 Reverse Link," IEEE Transactions on Vehicular Technology, vol. 49, no. 2, pp. 448-456, Mar. 2000.

[10] P. Frenger, P. Orten, T. Ottosson, "Code-Spread CDMA Using Maximum Free Distance Low-Rate Convolutional Codes," IEEE Transactions on Communications, vol. 48, no. 1, pp. 135-144, Jan. 2000.

[11] O. K. Tonguz, M. M. Wang, "Cellular CDMA Networks Impaired by Rayleigh Fading: System Performance with Power Control," IEEE Transactions on Vehicular Technology, vol. 43, no. 3, pp. 515-527, Aug. 1994.

[12] L. B. Milstein, T. S. Rappaport, R. Barghouti, "Performance Evaluation for Cellular CDMA," IEEE Journal on Selected Areas in Communications, vol. 10, no. 4, pp. 680-689, May 1992.

[13] S. W. Oh, K. L. Cheah, K. H. Li, "Forward-Link BER Analysis of Asynchronous Cellular DS-CDMA over Nakagami-Faded Channels Using Combined PDF Approach," IEEE Transactions on Vehicular Technology, vol. 49, no. 1, pp. 173- 180, Jan. 2000.

[14] Frankie Mak, "Cellular IS-95 CDMA Forward Link Simulator" university of Queensland, Oct.2002

[15] Yang Qi Shi Haoshan, Han Zhongxiang, "Analysis of BER for Mc-CDMA with effect of Multipath" China, Vol.2, pp 136-140, ICEMI 2007. 